\title{
EVALUATION OF RIFAXIMIN IN MANAGEMENT OF HEPATIC ENCEPHALOPATHY
}

\author{
By \\ ALI A. WAHIB ${ }^{1}$, M. NEGM EL-DEEN SALEM ${ }^{1}$, MOSTAFA A. AHMED ${ }^{1}$, \\ YASSER M. M. EL-DESSOUKY ${ }^{1}$, DIAA M. EL-TIBY ${ }^{1}$, KHALED EL-MOLA ${ }^{1}$ \\ AND ABEER M. EL-SAYED ${ }^{2}$ \\ By
}
Department of Tropical Medicine ${ }^{1}$, Faculty of Medicine, Al-Azhar University, Nasr City and Department of Pharmacognosy ${ }^{2}$, Faculty of Pharmacy, Cairo University, Giza, Egypt

\section{Abstract}

This study evaluated the efficacy of rifaximin versus lactulose in the treatment of hepatic encephalopathy (HE). The study population included 50 patients who were diagnosed to have signs of the first to third degree HE, according to the West Haven criteria and classified into two groups. GI: included 25 patients who had HE and were treated with lactulose syrup (laxolac) $90 \mathrm{ml}$ daily divided into 3 doses for 7 days. GII: included 25 patients who had HE and were treated with rifaximin $1200 \mathrm{mg}$ daily divided into 3 doses for 7 days. Patients in both groups also received other measures of treatment of $\mathrm{HE}$ as daily enema and protein restriction. Rifaximin significantly improved various subjective and measurable components of HE including mental status, behavior, asterixis, and serum ammonia concentration. In acute HE of grade 1 to grade 3, rifaximin may be a good alternative to non-absorbable disaccharides as it is broad spectrum, non-absorbable antibiotic with rapid action and little side effects. It is better tolerated, associated with less frequent and shorter hospitalization in comparison to lactulose.

Key words: Hospitalized patients, Hepatic encephalopathy, Lactulose, Rifaximin.

\section{Introduction}

Hepatic encephalopathy (HE) is a reversible neuropsychiatric complication that can develop in patients with severe acute or chronic liver disease (Christiansen et al, 2014). It is the second most common major complication in cirrhotic, following ascites (Zullo et al, 2012). It is a common complication of patients with hepatic cirrhosis and results in the alteration of mental status with a broad range of signs and symptoms based on its clinical severity. The presence of overt encephalopathy carries a poor prognosis with less than a $50 \%$ survival at one year after its initial development (Zetterman, 2012).

Although different factors have been implicated in HE pathogenesis, including mercaptans, short-chain fatty acids amines, $\gamma$ aminobutyric acid, endorphins, glutamate, endogenous benzodiazepine agonists, tryptophan, zinc deficiency, manganese deposition in the basal ganglia and indole (Riggio et al, 2005), plasma ammonia certainly remains the key factor (Prakash and Mullen, 2012).

Elevated serum ammonia level is the best described cause of $\mathrm{HE}$ and is detected in $60 \%-80 \%$ of affected patients. Current treatment strategies are aimed at reducing the serum level of ammonia. This is done by introducing agents that reduce or inhibit production of intestinal ammonia or minimize its absorption from the gastrointestinal tract as well as correcting any detectable precipitating factors (Eltawil et al, 2012).

Ammonia is mainly produced in the gut by glutamine metabolism in the small bowel and by bacterial flora in the large bowel. Therefore, current therapeutic approaches for $\mathrm{HE}$ treatment and prevention mainly rely on ammonia lowering strategies (Prakash and Mullen, 2012).

Unfortunately, recurrence of $\mathrm{HE}$ is not a rare event in these patients, even in the absence 
of any identifiable precipitating factor. Different therapeutic approaches have been attempted to prevent HE recurrence, such as branched-chain amino acids supplementation, acetyl-1-carnitine, sodium benzoate, zinc, acarbose and ornithine aspartate, with conflicting results in term of efficacy and possible side-effects (Schmid et al, 2010).

In clinical practice, administration of nonabsorbable disaccharides is commonly applied to both treat and prevent HE in patients with advanced disease or in cirrhotic at increased risk, such as those with transjugular intrahepatic portosystemic shunt (TIPS) (Morgan and Amodio, 2005). Indeed, both lactulose and lactitol administered oral (or by enema when patient is in coma) are able to reduce both the production and absorption of ammonia through different mechanisms. At the standard doses used $(30-60 \mathrm{mg} / \mathrm{d})$, non-absorbable disaccharides therapy is safe, generally well tolerated, effective in both treating and preventing an overt HE episode, including the minimal HE (Prasad et al, 2007). Therefore, this corner stone therapy is generally considered as the current golden standard for comparison with all other therapeutic strategies (Riggio et al, 2010).

A new semisynthetic antibiotic molecule belonging to the rifamycin derivatives, namely rifaximin, was synthesized in 1982 in Italy (Gupta et al, 2010). Rifaximin (4deoxy-4'-methylpyrido-(1',2'-1,2)-imidazo(5,4C)-rifamycin SV) acts by inhibiting bacterial ribonucleic acid (RNA) synthesis. Rifaximin is virtually unabsorbed after oral administration and exhibits broad spectrum antimicrobial activity against both aerobic and anaerobic gram-positive and gramnegative microorganisms within the gastrointestinal tract. In healthy individuals, as much as $96 \%$ of radiolabeled rifaximin was recovered in the stool and only $0.32 \%$ in the urine (Bajaj and Riggio, 2010).

Different authors investigated the efficacy of rifaximin therapy in both short-term management of acute HE episodes and in long- term therapy for prevention of recurrence. In a systematic review, rifaximin has been found to be at least equally effective or superior to non-absorbable disaccharides and antimicrobials in relieving signs or symptoms observed in patients with mild or moderately severe HE (Lawrence and Klee, 2008). Besides, a reduced risk of variceal hemorrhage, HE, spontaneous bacterial peritonitis, hepatorenal syndrome, and thrombocytopenia follows long-term use of rifaximin (Zetterman, 2012).

\section{Patients and Methods}

This study was carried out at Tropical Medicine Department (Al-Hussein and Sayed Galal university Hospitals). The study population included 50 patients who were diagnosed to have signs of the first to third degree HE, according to the West Haven criteria suggested according to Blei and Cordoba (2001) and classified into two groups: GI: included 25 patients who had HE and were treated with lactulose syrup (laxolac) $90 \mathrm{ml}$ daily divided into 3 doses for 7 days as suggested by Cash et al. (2010). GII: included 25 patients who had $\mathrm{HE}$ and were treated with rifaximin $1200 \mathrm{mg}$ daily divided into 3 doses for 7 days as suggested by Williams et al. (2000) and Festi et al. (2006).Besides, patients of both groups also received other measures of treatment of hepatic encephalopathy as daily enema and protein restriction.

Inclusion criteria: Patients showing signs of the first to third degree HE according to the West Haven criteria and have elevated blood ammonia level and randomly divided into two groups.

Exclusion criteria: 1-Presence of a major neuropsychiatric illness. 2- Presence of intestinal obstruction or inflammatory bowel disease. 3- A serum creatinine level $>$ twice normal. 4- Patients with GIT bleeding. 5Patients with spontaneous bacterial peritonitis (S.B.P).

All patients were subjected to: 1- Full history taking: (from the patient or his relatives) focusing on the number of attacks and the 
possible precipitating factors. 2- Detailed clinical examination including: General examination: to detect the grade of mental state according to West Haven criteria, signs of liver cell failure and grade of flapping tremors.

A. Flapping tremors were graded according to Yong-Han et al (2005) as follows: Grade 0: no tremors. Grade 1: few flapping motions. Grade 2: occasional flapping motions. Grade 3: frequent flapping motions and Grade 4: almost continuous flapping motions.

B. Grading of mental state according to West Haven classification system (Blei and Cordoba, 2001). Grade 0: Minimal HE (previously known as subclinical HE): Lack of detectable changes in personality or behavior; Minimal changes in memory, concentration, intellectual function, and coordination and no Asterixis. Grade 1: Trivial lack of awareness, shortened attention span, impaired addition or subtraction, hypersomnia, insomnia, or inversion of sleep pattern; Euphoria, depression, or irritability. Mild confusion and slowing of ability to perform mental tasks; Asterixis can be detected. Grade 2: Lethargy or apathy, disorientation, inappropriate behavior, slurred speech, drowsiness, gross deficits in ability to perform mental tasks, obvious personality changes and intermittent disorientation, usually regarding time and obvious of Asterixis. Grade 3: Somnolent but can be aroused, unable to perform mental tasks, disorientation about time and place, marked confusion, amnesia, occasional fits of rage. Grade 4: Coma with or without response to painful stimuli.

Grade of flapping tremors and grade of mental state were done before beginning the treatment in the first day and then on days 3 , $5 \& 7$ of the trial period for both groups.

Number connection test (NCT): Number connection test is the time taken to connect 25 progressive numbers. The test was done for forty patients only. It was determined pretreatment and post treatment for both groups (on days 1 and 7 of treatment). $\mathrm{Pa}$ tients with gross disorientation were excluded from the test (10 patients). It was graded as follows (Paik et al, 2005): Grade 0: $<30$ sec (normal).Grade 1: 31-50 sec. Grade 2: 51-80 sec. Grade 3: 81-120 sec. Grade 4: > $120 \mathrm{sec}$.

Routine investigations (done in days $1 \& 7$ of treatment) including: 1- Urine analysis. 2Co-mplete blood count (CBC). 3- Liver function tests: ALT, AST, total protein, serum albumin, serum bilirubin, (prothrombin time, prothrombin concentration, INR). 4Kidney function tests: blood urea and serum creatinine. 5- Viral Markers: HCVAb and HBsAg. 6- K and Na level. 7- Ascitic fluid analysis: if ascites is detected to exclude SBP. 8- Serum ammonia level:

Blood samples were collected from a stasis-free vein (that is, without using a tourniquet and taking care not to cause turbulence or hemolysis) and immediately transported on ice to the laboratory to be analyzed within $20 \mathrm{~min}$. Normal ammonia level was considered $<75 \mathrm{umol} / \mathrm{L}$. It was measured before and after treatment (on days $1 \& 7$ of treatment) according to Yong-Han et al (2005) and graded as follows: Grade $0:<75 \mu \mathrm{M} / \mathrm{L}$, Grade 1:76-150 $\mu \mathrm{M} / \mathrm{L}$, Grade 2: 151-200 $\mu \mathrm{M} / \mathrm{L}$, Grade 3:201-250 $\mu \mathrm{M} / \mathrm{L}$ and Grade 4: $>251 \mu \mathrm{M} / \mathrm{L}$

Hepatic encephalopathy (HE) index: Grade for the mental state, grade of flapping tremors, grade of NCT plus blood ammonia level grade were weighted in proportion to their importance. Mental status grade awarded a weighting factor of three, while the other variables were each assigned a factor of one. HE index was defined as the total of the weighted grades, and had a possible range of 0 to 23 points. It was calculated before and after treatment (on days $1 \& 7$ of treatment) for both groups (Paik et al, 2005). HE index $=($ grade of mental state $) \times 3+$ (grade of number connection test) + (grade of flapping tremor) + (grade of blood ammonia). 
A decrease of HE index by at least one point was defined as improved, and incre-

Imaging evaluation: 1-Chest $\mathrm{x}$-ray to exclude chest infection, 2- Pelvi-abdominal ultrasonography.

Statistical analysis: Results were tabulated and statistical analysis was done and the results were considered significant at $\mathrm{P}$ value ment of the HE index by one point or more was defined as worsened (Paik et al, 2005). $<0.05$. Statistical presentation and analysis of the present study was conducted, using the mean, standard deviation, student t-test, Paired t-test, Chi-square and Mann-Whitney by SPSS V17.

\section{Results}

Table 1: Precipitating factors of HE in both groups

\begin{tabular}{|l|c|c|c|c|}
\hline \multicolumn{2}{|c}{ precipitating factors } & Lactulose $(\mathrm{n}=25)$ & Rifaximin $(\mathrm{n}=25)$ & Total $(\mathrm{n}=50)$ \\
\hline \multirow{2}{*}{ Protein overload } & No. & 9 & 15 & 24 \\
\cline { 2 - 5 } Infection & $\%$ & 36.00 & 60.00 & 48.00 \\
\hline \multirow{2}{*}{ Constipation } & No. & 9 & 3 & 12 \\
\cline { 2 - 5 } & $\%$ & 36.00 & 12.00 & 24.00 \\
\hline \multirow{2}{*}{ Unknown precipitating factor } & No. & 3 & 2 & 5 \\
\cline { 2 - 5 } & $\%$ & 12.00 & 5.00 & 10.00 \\
\hline \multirow{2}{*}{ Total } & No. & 4 & 20.00 & 18.00 \\
\hline \multirow{2}{*}{ Chi-square } & No. & 16.00 & 25 & 100.00 \\
\cline { 2 - 5 } & $\%$ & 100.00 & 0.372 & \\
\hline
\end{tabular}

Table 2: Main level of blood ammonia $(\mu \mathrm{mol} / \mathrm{l})$ pre $\&$ post treatment in both groups

\begin{tabular}{|c|c|c|c|c|c|c|c|c|}
\hline \multirow{3}{*}{ Group } & \multicolumn{4}{|c|}{ Blood ammonia $(\mu \mathrm{mol} / \mathrm{l})$} & \multicolumn{2}{c|}{ Paired t-test } \\
\cline { 2 - 9 } & \multicolumn{2}{|c|}{ Pre treatment } & \multicolumn{2}{c|}{ Post treatment } & \multicolumn{2}{c|}{} \\
\cline { 2 - 9 } & Mean & \pm & SD & Mean & \pm & SD & T & P-value \\
\hline Lactulose & 175.880 & \pm & 51.386 & 136.560 & \pm & 40.508 & 9.430 & 0.000 \\
\hline Rifaximin & 179.400 & \pm & 19.570 & 135.760 & \pm & 21.423 & 14.068 & 0.000 \\
\hline
\end{tabular}

Table 3: Changes in grading of flapping tremors in both groups in $1^{\text {st }}, 3^{\text {rd }}, 5^{\text {th }} \& 7^{\text {th }}$ days of treatment

\begin{tabular}{|c|c|c|c|c|c|c|c|c|c|}
\hline \multirow{2}{*}{\multicolumn{2}{|c|}{ Flapping tremors }} & \multicolumn{2}{|c|}{ Lactulose $(n=25)$} & \multicolumn{2}{|c|}{$\operatorname{Rifaximin}(n=25)$} & \multicolumn{2}{|c|}{ Total $(n=50)$} & \multicolumn{2}{|c|}{ Mann-Whitney Test } \\
\hline & & No. & $\%$ & No. & $\%$ & No. & $\%$ & $\mathrm{Z}$ & $\mathrm{P}$-value \\
\hline \multirow{5}{*}{ After 1day } & G0 & 0 & 0.00 & 0 & 0.00 & 0 & 0.00 & \multirow{5}{*}{-0.622} & \multirow{5}{*}{0.534} \\
\hline & G1 & 1 & 4.00 & 0 & 0.00 & 1 & 2.00 & & \\
\hline & G2 & 6 & 24.00 & 9 & 36.00 & 15 & 30.00 & & \\
\hline & G3 & 16 & 64.00 & 15 & 60.00 & 31 & 62.00 & & \\
\hline & G4 & 2 & 8.00 & 1 & 4.00 & 3 & 6.00 & & \\
\hline \multirow{5}{*}{ After 3days } & G0 & 0 & 0.00 & 9 & 36.00 & 9 & 18.00 & \multirow{5}{*}{-4.544} & \multirow{5}{*}{0.000} \\
\hline & G1 & 3 & 12.00 & 6 & 24.00 & 9 & 18.00 & & \\
\hline & G2 & 10 & 40.00 & 10 & 40.00 & 20 & 40.00 & & \\
\hline & G3 & 12 & 48.00 & 0 & 0.00 & 12 & 24.00 & & \\
\hline & G4 & 0 & 0.00 & 0 & 0.00 & 0 & 0.00 & & \\
\hline \multirow{5}{*}{ After 5days } & G0 & 1 & 4.00 & 15 & 60.00 & 16 & 32.00 & \multirow{5}{*}{-4.424} & \multirow{5}{*}{0.000} \\
\hline & G1 & 6 & 24.00 & 5 & 20.00 & 11 & 22.00 & & \\
\hline & $\mathrm{G} 2$ & 13 & 52.00 & 5 & 20.00 & 18 & 36.00 & & \\
\hline & G3 & 4 & 16.00 & 0 & 0.00 & 4 & 8.00 & & \\
\hline & G4 & 1 & 4.00 & 0 & 0.00 & 1 & 2.00 & & \\
\hline \multirow{5}{*}{ After 7days } & G0 & 3 & 12.00 & 18 & 72.00 & 21 & 42.00 & \multirow{5}{*}{-4.000} & \multirow{5}{*}{0.000} \\
\hline & G1 & 12 & 48.00 & 4 & 16.00 & 16 & 32.00 & & \\
\hline & G2 & 7 & 28.00 & 3 & 12.00 & 10 & 20.00 & & \\
\hline & G3 & 2 & 8.00 & 0 & 0.00 & 2 & 4.00 & & \\
\hline & G4 & 1 & 4.00 & 0 & 0.00 & 1 & 2.00 & & \\
\hline
\end{tabular}


Table 4: Changes in grading of mental status in both groups in $1^{\text {st }}, 3 \mathrm{rd}, 5^{\text {th }} \& 7^{\text {th }}$ of treatment.

\begin{tabular}{|c|c|c|c|c|c|c|c|c|c|}
\hline \multirow{2}{*}{\multicolumn{2}{|c|}{ Grade of mental status }} & \multicolumn{2}{|c|}{ Lactulose $(n=25)$} & \multicolumn{2}{|c|}{ Rifaximin $(n=25)$} & \multicolumn{2}{|c|}{ Total $(n=50)$} & \multicolumn{2}{|c|}{ Mann-Whitney Test } \\
\hline & & No. & $\%$ & No. & $\%$ & No. & $\%$ & $\mathrm{Z}$ & P-value \\
\hline \multirow{4}{*}{ After 1day } & G0 & 0 & 0.00 & 0 & 0.00 & 0 & 0.00 & \multirow{4}{*}{-0.341} & \multirow{4}{*}{0.733} \\
\hline & G1 & 3 & 12.00 & 6 & 24.00 & 9 & 18.00 & & \\
\hline & G2 & 15 & 60.00 & 11 & 44.00 & 26 & 52.00 & & \\
\hline & G3 & 7 & 28.00 & 8 & 32.00 & 15 & 30.00 & & \\
\hline \multirow{4}{*}{ Afetr 3days } & G0 & 1 & 4.00 & 12 & 48.00 & 13 & 26.00 & \multirow{4}{*}{-4.037} & \multirow{4}{*}{0.000} \\
\hline & G1 & 10 & 40.00 & 10 & 40.00 & 20 & 40.00 & & \\
\hline & G2 & 11 & 44.00 & 3 & 12.00 & 14 & 28.00 & & \\
\hline & G3 & 3 & 12.00 & 0 & 0.00 & 3 & 6.00 & & \\
\hline \multirow{4}{*}{ After 5days } & G0 & 1 & 4.00 & 19 & 76.00 & 20 & 40.00 & \multirow{4}{*}{-4.974} & \multirow{4}{*}{0.000} \\
\hline & G1 & 16 & 64.00 & 5 & 20.00 & 21 & 42.00 & & \\
\hline & G2 & 7 & 28.00 & 1 & 4.00 & 8 & 16.00 & & \\
\hline & G3 & 1 & 4.00 & 0 & 0.00 & 1 & 2.00 & & \\
\hline \multirow{4}{*}{ After 7days } & G0 & 8 & 32.00 & 21 & 84.00 & 29 & 58.00 & \multirow{4}{*}{-3.559} & \multirow{4}{*}{0.000} \\
\hline & G1 & 14 & 56.00 & 3 & 12.00 & 17 & 34.00 & & \\
\hline & G2 & 2 & 8.00 & 1 & 4.00 & 3 & 6.00 & & \\
\hline & G3 & 1 & 4.00 & 0 & 0.00 & 1 & 2.00 & & \\
\hline
\end{tabular}

Table 5: Hepatic Encephalopathy Index (HE index) pre \& post treatment in both groups

\begin{tabular}{|c|c|c|c|c|c|c|c|c|c|}
\hline \multirow{2}{*}{ Group } & \multicolumn{5}{|c|}{ HE INDEX } & \multicolumn{2}{c|}{ T-test } \\
\cline { 3 - 10 } & \multicolumn{3}{|c|}{ Range } & Mean & \pm & SD & t & P-value \\
\hline \multirow{2}{*}{ Pre } & Lactulose & 6.00 & - & 23.00 & 13.840 & \pm & 4.696 & \multirow{2}{*}{0.264} & \multirow{2}{*}{0.793} \\
\cline { 2 - 10 } & Rifaximin & 8.00 & - & 20.00 & 13.560 & \pm & 2.451 & & \multirow{2}{*}{0.006} \\
\hline \multirow{2}{*}{ Post } & Lactulose & 3.00 & - & 16.00 & 7.720 & \pm & 3.747 & \multirow{2}{*}{2.881} & \multirow{2}{*}{0.006} \\
\cline { 2 - 7 } & Rifaximin & 3.00 & - & 13.00 & 4.920 & \pm & 3.095 & &
\end{tabular}

\section{Discussion}

$\mathrm{HE}$ is a metabolically induced, potentially reversible, functional disturbance of the brain. While HE may be a complication of acute or chronic liver disease, it is most commonly associated with cirrhosis (Mas, 2006).

Depending on its cause, HE can be categorized as either type $\mathrm{A}$, which occurs in patients with acute liver failure; type B, which occurs in patients with bypass shunts or type $\mathrm{C}$, which occurs in patients with chronic liver disease (Frederick, 2011).

The pathophysiology of HE is complex and it manifests with progressive deterioration of the superior neurological functions. HE occurs in the presence of insufficient hepatic clearance of toxins absorbed from the intestine resulting in neurochemical abnormalities across the blood brain barrier. The clinical manifestations of HE range from altered mental status to deep coma (Eltawil et al, 2012).
Elevated serum ammonia level is the best described cause of $\mathrm{HE}$ and is detected in $60 \%-80 \%$ of affected patients. Current treatment strategies are aimed at reducing the serum level of ammonia. This is done by introducing agents that reduce or inhibit production of intestinal ammonia or minimize its absorption from the gastrointestinal tract as well as correcting any detectable precipitating factors (Eltawil et al, 2012).

Traditionally, non-absorbable disaccharides have been used as the first-line therapy for patients with HE. Although safe, the need to adjust disaccharide doses to achieve two to three loose bowel movements per day often leads to frequent nausea, vomiting, and flatulence and affects compliance (Phongsamran et al, 2010).

On the other hand, rifaximin is an agent that appears to be effective in the treatment of HE without carrying the risk of severe side effects. It has the advantage of being well tolerated and has minimal risk of causing 
bacterial resistance. It was initially introduced in Italy in 1987and recently approved in the United States for the treatment and prevention of HE. The purported advantages of rifaximin over other oral agents make it a very attractive choice for treatment of $\mathrm{HE}$, although at considerably greater expense (Phongsamran et al, 2010).

In this study the cause of the liver disease was hepatitis $\mathrm{C}$ virus (HCV) infection in $100 \%$ of cases in both groups. In Egypt, the main cause of decompensated liver disease is HCV infection, because Egypt developed the world's highest rates of HCV infection over a short period of time (Strickland, 2006).

Regarding age and sex distribution there was no statistically significant difference between both groups.

Encephalopathy was presented for the first time in 4/25(16\%) in GI versus 5/25(20\%) in GII with no significant differences between both groups. This finding means that most of patients presented by recurrent attacks of HE. This meets the finding of Bajaj et al (2010) who found that many patients suffer from intermittent or episodic HE. Episodes of HE may be isolated events, but more commonly they are recurrent, with patients having seemingly normal cognitive function between episodes.

In this study, protein overload was the precipitating factor in $9 / 25(36 \%)$ patients in GI \& 15/25(60\%) patients in GII. Infection such as (chest infection, urinary tract infection or gastroenteritis) was present in $9 / 25$ patients in GI \& $3 / 25$ patients in GII. Constipation was present in $3 / 25$ patients in GI \& $2 / 25$ patients in group II. Four from 25 patients in GI \& 5/25 patients in GII were presented with unknown precipitating factor of $\mathrm{HE}$, which means that protein overload was the most common precipitating factor for HE.

These data agreed with Cash et al. (2010), Chung and Podolsky (2005) and Sundaram and Shaikh (2009), who reported that consumption of large amounts of protein was one of the common precipitating factor of
$\mathrm{HE}$ as it led to increase ammonia level in blood which is the most widely accepted theory for the pathogenesis of HE (EASL, 2011). Also Yong-Han et al (2005) reported in their study that $8 / 32(25 \%)$ of patients presented by HE was precipitated by protein over load. But Antoni et al. (2003) reported in that comparison between rifaximin and lactitol that only $1(2 \%)$ in rifaximin group $\& 1(1.9 \%)$ in lactitol group were precipitated by protein overload.

In this study $4 / 25(16 \%)$ of patients in GI and $5 / 25(5 \%)$ of patients in GII were presented with unknown precipitating factor of hepatic encephalopathy. That was similar to the findings of Cash et al. (2010) who found that in some cases, no clear cause for an attack of HE can be found. Also, Yong-Han et al (2005) concluded that $7(21.9 \%)$ patients in rifaximin group \& $5(22.9 \%)$ patients in lactulose group were presented by $\mathrm{HE}$ of unknown precipitating factor. So, many patients suffer from episodic HE, with episodes being either precipitated or spontaneous (Bajaj et al, 2010).

Regarding laboratory investigations (hemoglobin level, platelet count, serum creatinine, ALT level, AST level, serum albumin, and bilirubin level) there was no significant difference between both groups pre and post treatment.

Regarding blood ammonia level, there was a very high significant statistical difference between pretreatment and post treatment levels in GI (lactulose) with $\mathrm{p}<0.001$. Also, there was a very high significant statistical difference between pretreatment and post treatment levels in GII (rifaximin) with $p<0.001$. But, in rifaximin group the decrease in ammonia level was more than in the lactulose group. These results were similar to that Yong-Han et al. (2005) and Mas (2006).

This supports that increase ammonia level in the blood is the most widely accepted theory of the pathogenesis of HE, and ithought to be a central player in the development of HE (EASL, 2011). 
Both rifaximin and lactulose can decrease ammonia level by different mechanisms but rifaximin is more rapid than lactulose. This can be explained by the fact that rifaximin acts by inhibiting bacterial ribonucleic acid (RNA) synthesis and it is unabsorbed after oral administration and exhibits broad spectrum antimicrobial activity against both aerobic and anaerobic, gram-positive and gram-negative microorganisms within the gastrointestinal tract (Scapignato and Pelosini, 2006), so, rifaximin lowers the bacterial load 1001000 folds (Darkoh et al, 2010).

Regarding the grading of flapping tremors, the results showed that after 3 days of treatment with rifaximin 9 patients had no flapping tremors and this number increased to 18 patients after the end of treatment. But as regard lactulose group no patients became in grade 0 flapping tremors after 3 days of treatment and only 3 patients had no flapping tremors after end of treatment. These results showed that the response of patients regarding improvement of flapping tremors was more \& rapid in rifaximin group than lactulose group. These data agreed with Neff et al (2009) who reported that the response to rifaximin ranged from $80 \%$ to $90 \%$, with a faster onset of action by up to 3 days. Moreover, Loguercio et al. (2003) documented faster improvement of asterixis in patients treated with rifaximin. As $80 \%$ to $90 \%$ of rifaximin taken by mouth concentrates in the gut, this accumulation enhances the effectiveness of rifaximin in the treatment of intestinal infections. Due to its distribution in the gut, rifaximin is excreted almost entirely in the feces and its half-life is approximately six hours, so its action more rapid than lactulose.

As regard grading of mental status, the results showed that after 3 days, 12 patients became in grade 0 in the group treated with rifaximin versus 1 patients only in the group treated with lactulose. After the end of treatment 21 patient $(84 \%)$ in rifaximin group became in grad
0 but only 8 patients (32\%) in lactulose group became in grade 0 . These results showed that the response of patients regarding improvement of mental status was more \& rapid in rifaximin group than lactulose group. These data agreed with Neff et al. (2009) who reported in their study that the response to rifaximin ranged from $80 \%$ to $90 \%$, with a faster onset of action by up to 3 days. Also Loguercio et al (2003) documented faster improvement in $\mathrm{HE}$ and higher percentages of normalized state in patients treated with rifaximin. Also, these results are similar to that reported by Bucci and Palmieri (1993) who found that the improvement in the rifaximin group was significantly superior to that of lactulose group as regard mental status.

Also, Mas (2006) reported in that improvement in grading of mental status for rifaximin was superior to lactitol. Moreover, two trials confirmed that it is a useful alternative to disaccharides in patients with grades I - III HE in improving mental status (Williams et al, 2000; Festi et al, 2006). Moreover, Hina et al. (2010) reported that rifaximin was effective in improving behavioral, laboratory, mental status and intellectual abnormalities associated with hepatic encephalopathy and patients treated with rifaximin also required shorter duration of hospitalization compared with lactulose. It may be due to that rifaximin is unabsorbed by the gut, thereby allowing the antibiotic to reach high concentrations in the intestinal tract and to remain in the feces in its active form. Due to these properties (high antibacterial activity with null absorption), rifaximin decreases plasma ammonia levels and improves the symptoms related to $\mathrm{HE}$ rapidly.

As regard to the HE index the results showed that the improvement was more and rapid in rifaximin group than lactulose group with statistically significant difference between the two groups post treatment at $p$ 
value $<0.05$ (table 5). This means that the total and global effect of rifaximin was better and more effective than lactulose.

\section{Conclusions}

Rifaximin significantly improved the various subjective and measurable components of hepatic encephalopathy including mental status, behavior, asterixis, and serum ammonia concentration. In acute $\mathrm{HE}$ of grade 1 to grade 3 , rifaximin may be a good alternative to non-absorbable disaccharides as it is broad spectrum and non-absorbable antibiotic with rapid action and little side effects. It is also better tolerated and associated with less frequent and shorter hospitalization in comparison to lactulose.

Thus, Rifaximin must be used in addition to the enema because enema is the corner stone in the treatment of HE.

\section{References}

Antoni, M, Juan, R, Lourdes, S, et al, 2003: Comparison of rifaximin and lactitol in the treatment of acute hepatic encephalopathy: results of a randomized, double-blind, doubledummy, controlled clinical trial. Gastroenterology 140:478-87.

Bajaj, JS, Riggio, O, 2010: Drug therapy: rifaximin. Hepatology 52:1484-8.

Bajaj, JS, Schubert, CM, Heuman, DM, et al, 2010: Persistent cognitive impairment after resolution of overt encephalopathy. Gastroenterology 138:2332-40.

Blei, AT, Cordoba, J, 2001: Hepatic Encephalopathy. Am. J. Gastroenterol. 96, 7:1968-76.

Bucci, L, Palmieri, GC, 1993: Double-blind, double-dummy comparison between treatment with rifaximin and lactulose in patients with medium to severe degree of hepatic encephalopathy. Curr. Med. Res. Opin. 13:109-18.

Cash, WJ, McConville, P, McDermott, E, et al, 2010: Current concepts in the assessment and treatment of hepatic encephalopathy. QJM 103, 1:9-16.

Christiansen, KM, Mössner, BK, Hansen, JF, Jarnbjer, EF, Pedersen, C, et al, 2014: Liver stiffness measurement among patients with chronic hepatitis B and C: Results from a 5-year prospective study. PLoS One 9, 11:e111912

Chung, RT, Podolsky, DK, 2005: Cirrhosis and its complications. In: Harrison's Principles of Internal Medicine. $16^{\text {th }}$ ed. NY: McGraw-Hill.
Darkoh, C, Lichtenberger, LM, Ajami, N, et al, 2010: Bile acids improve antimicrobial effect of rifaximin. Antimicrobial. Agents Chemother. 54: 3618-24.

EASL, 2011: Treatment of patients with hepatic encephalopathy: Review of the Latest Data. Gastroenterol. hepatol. 7, 6:1-16.

Eltawil, KM, Laryea, M, Peltekian, K, et al, 2012: Rifaximin vs. conventional oral therapy for hepatic encephalopathy: A meta-analysis. Wld. J. Gastroenterol. 18, 8:767-77.

Festi, D, Vestito, A, Mazzella, G, et al, 2006: Management of hepatic encephalopathy: focus on antibiotic therapy. Digestion 73, 1:94-101.

Frederick, RT, 2011: Current concepts in the pathophysiology and management of hepatic encephalopathy. Gastroenterol. Hepatol. 7:222-33.

Gupta, A, Dhiman, RK, Kumari, S, et al, 2010: Role of small intestinal bacterial overgrowth and delayed gastrointestinal transit time in cirrhotic patients with minimal hepatic encephalopathy. J. Hepatol. 53:849-55.

Hina, Y, Wafa, BS, Khawaja, TM, 2010: Identification, prevention and management of risks associated with hepatic encephalopathy. J. Pharmaceut. Sci. Res. 2, 12:814-20.

Lawrence KR, Klee JA, 2008: Rifaximin for the treatment of hepatic encephalopathy. Pharmacotherapy 28:1019-32.

Loguercio, C, Federico, A, De Girolamo, V, et al, 2003: Treatment of chronic hepatic encephalopathy with rifaximin: Results of a double-blind clinical study. Minerva Gastroenterol. Dietol. 49:53-62.

Mas, A, 2006: Hepatic encephalopathy: from pathophysiology to treatment. Digestion 73,1 : 86-93.

Morgan, MY, Amodio, P, 2005: Treatment for hepatic encephalopathy: tips from TIPS? J. Hepatol. 42:626-8.

Neff, G, Kenimer, N, Gaddis, A, et al, 2009: Efficacy of rifaximin in maintenance of remission in patients with hepatic encephalopathy. Am. J. Gastroenterol. 104:A415

Paik, YH, Lee, KS, Han. KH, et al, 2005: Comparison of rifaximin and lactulose for the treatment of hepatic encephalopathy: A Prospective Randomized Study. Yonsei Med. J. 46, 3: 399-407.

Phongsamran, PV, Kim, JW, Abbott, JC, et al, 2010: Pharmacotherapy for hepatic encephalopathy. Drugs 70:1131-48. 
Prakash, R, Mullen, KD, 2012: Mechanisms, diagnosis and management of hepatic encephalopathy. Nat. Rev. Gastroenterol. Hepatol. 7:515-25

Prasad, DS, Dhiman, RK, Duseja, A, et al, 2007: Lactulose improves cognitive functions and health-related quality of life in patients with cirrhosis who have minimal hepatic encephalopathy. Hepatology 45:549-59.

Riggio, O, Efrati, C, Catalano, C, et al, 2005: High prevalence of spontaneous portal systemic shunts in persistent hepatic encephalopathy: a case-control study. Hepatology 42, 5: 115-65.

Riggio, O, Mannaioni, G, Ridola, L, et al, 2010: Peripheral and splanchnic indole and oxindole levels in cirrhotic patients: a study on the pathophysiology of hepatic encephalopathy. Am. J. Gastroenterol. 105:1374-81.

Scarpignato, C, Pelosini, I, 2006: Experimental and clinical pharmacology of rifaximin, a gastrointestinal selective antibiotic. Digestion 73, 11:13-27.

Schmid, M, Peck-Radosavljevic, M, König, F, et al, 2010: A double-blind, randomized, placebo-controlled trial of intravenous L-ornithine-L- aspartate on postural control in patients with cirrhosis. Liver Int. 30, 4:574-82.

Strickland, GT, 2006: Liver disease in Egypt: hepatitis $\mathrm{C}$ superseded Schistosomiasis as a result of iatrogenic and biological factors. Hepatology 43:915-22.

Sundaram, V, Shaikh, OS, 2009: Hepatic encephalopathy: pathophysiology and emerging therapies. Med. Clin. North Am. 93, 4:819-36.

Williams, R, James, OF, Warnes TW, et al, 2000: Evaluation of the efficacy and safety of rifaximin in the treatment of hepatic encephalopathy: a double-blind, randomized, dose-finding multi-centre study. Eur. J. Gastroenterol. Hepatol.12:203-8.

Yong-Han, P, Kwan, SL, Kwang-Hyub, H, et al, 2005: Comparison of rifaximin and lactulose for the treatment of hepatic encephalopathy. Yonsei Med. J. 46, 3:399-407.

Zetterman, RK, 2012: Hepatic Encephalopathy: Association of West Indian Gastroenterologists. April, 2012.

Zullo, A, Hassan, C, Ridola, L, et al, 2012: Rifaximin therapy and hepatic encephalopathy: Pros and cons. Wld. J. Gastrointest. Pharmacol. Ther.

3 , $4: 62-7$. 